\title{
Cerebral Mechanism of Tuina Analgesia in Management of Knee Osteoarthritis using multimodal MRI: Study Protocol for a Randomized Controlled Trial
}

\section{Guangxin Guo}

Shanghai University of Traditional Chinese Medicine https://orcid.org/0000-0002-2081-5229

\section{Yazhuo Kong}

Institute of Psychology Chinese Academy of Sciences

\section{Qingguang Zhu}

Istitute of Tuina, Yueyang Hospital of Integrated Traditional Chinese and Western Medicine, Shanghai Univerdity of Traditional Chinese Medicine

\section{Zhiwei Wu}

Institute of Tuina, Yueyang Hospital of Integrated Traditional Chinese and Western Medicine, Shanghai University of Traditional Chinese Medicine

\section{Shuaipan Zhang}

Shanghai University of TCM: Shanghai University of Traditional Chinese Medicine

\section{Wuquan Sun}

Shanghai University of Traditional Chinese Medicine Yueyang Hospital of Integrated Traditional Chinese Medicine and Western Medicine

\section{Yanbin Cheng}

Shanghai University of Traditional Chinese Medicine Yueyang Hospital of Integrated Traditional Chinese Medicine and Western Medicine

Min Fang ( $\nabla$ fangmin19650510@163.com )

Shanghai University of Traditional Chinese Medicine

\section{Study protocol}

Keywords: Knee Osteoarthritis, Chronic Pain, Tuina (Chinese massage), Analgesia Mechanism, Multimodal MRI

Posted Date: January 12th, 2021

DOl: https://doi.org/10.21203/rs.3.rs-75452/v1 
License: (c) (i) This work is licensed under a Creative Commons Attribution 4.0 International License. Read Full License 


\section{Abstract}

Background: Chronic pain of patients with knee osteoarthritis (KOA) seriously affects the quality of life and leads to the heavy social and economic burden. As one of the representative non-drug therapies in Traditional Chinese Medicine (TCM), Tuina has been generally recognized as safe and effective for reducing the chronic pain of $\mathrm{KOA}$, however the underlying central mechanisms of Tuina for improving the pain of KOA are not fully understood.

Methods/design: This study is a randomized controlled trial with parallel-group design. In total, 60 eligible participants will be randomly assigned to a Tuina group and a health care education group (Education group) in a 1:1 ratio with gender and age matched. The interventions of both groups will last for 30 min per session and be conducted twice each week for 12 weeks. This study will focus on detecting the changes of brain gray matter structure (GM), white matter structure (WM), the cerebral functional connectivity (FC) elicited by Tuina treatment, e.g thalamus, hippocampus, anterior cingulate gyrus, $\mathrm{S} 1$, insula, the periaqueductal gray subregions (PAG) etc. The two groups of patients will be evaluated by clinical assessments and multimodal magnetic resonance imaging (MRI) at the baseline and the end of 6 and 12 weeks' treatment, and still be evaluated by clinical assessments for 48 weeks of follow-up. The Visual Analog Scale (VAS) of current pain, Short-form McGill Pain Questionnaire (SF-MPQ), Western Ontario and McMaster Universities Osteoarthritis Index (WOMAC), the 36-Item Short Form Health Survey (SF-36), Hamilton Depression Scale (HAMD) and the Hamilton Anxiety Scale (HAMA) will be used to evaluate the pain intensity, pain feeling, pain emotion, clinical symptoms and quality of life respectively. The evaluators and statisticians will be blinded to the group allocation. The repeated measures analysis of variance ( 2 groups $\times 6$ time points ANOVA) will be employed to analyze numerical variables of the clinical and neuroimaging data obtained in the study. $P<0.05$ will be the statistical significance level.

Discussion: The results of this randomized controlled trial with clinical assessments and multimodal MRI will help to reveal the influence of Tuina treatment on the potential morphological changes of cortical and subcortical brain structures, the white matter integrity and the functional activities and connectivity of brain regions of patients with KOA, which might provide scientific evidence for the clinical application of Tuina in management of KOA.

Trial registration: Chinese Clinical Trial Registry: ChiCTR2000037966. Date of Registration: 8, Sep. 2020. http://www.chictr.org.cn/edit.aspx?pid=61083\&htm=4

\section{Background}

KOA is an important cause of knee pain and disability, which brings serious personal and social burden all over the world[1, 2]. The total prevalence of KOA is about $17.0 \%$ in recent years $[3,4]$. With the aging of the population, the prevalence of symptomatic KOA is increasing $[1,2,5,6]$. It is estimated that the elderly population will reach 480 million by 2050, and the negative impact caused by KOA pain will be more serious[4]. Most patients with KOA have long term chronic pain experience. Pain can run through the 
occurrence, development and outcome of KOA and occurs repeatedly, which seriously affected work and life. Symptomatic KOA can increase the risk of all-cause mortality among the residents in the rural areas[1, 7]. Pain, as a typical manifestation and the most common complaint of patients with KOA, has become a hot topic in clinical and scientific research[1].

The International Society for the Study of Pain (IASP) redefines pain as an unpleasant sensory and emotional experience associated with, or resembling that associated with, actual or potential tissue damage[8]. KOA pain is also multi-dimensional, which is produced by the peripheral and central levels of the nervous system, and the regulatory mechanism is complex. The manifestations of KOA chronic pain are complex and varied, such as persistent pain or intermittent pain, with or without neuropathological components, with or without central sensitization[9]. Previous study suggests that pain may be associated with the changes of the functional activity and structures of the cortical and subcortical brain regions in the acute and chronic pain[10].

Chronic pain of KOA is complex[11]. The brain plays a key role in the occurrence and relief of chronic pain as studies have shown brain plasticity changes (e.g. GM structure, WM structure and brain functional activities) after chronic pain using multimodal MRI. Central sensitization, as an important manifestation and pathological change of central nervous system, plays an important role in the pathogenesis of knee arthritis[12,13]. With the wide application of brain MRI technology in pain research, investigators tried to map the brain regions involved in the central pathogenesis of chronic pain, and partly explored the central mechanism of Tuina analgesia[14, 15]. The chronic and persistent pain as the defining symptom of KOA is closely related to pathological changes of cortical structure and subcortical brain nuclei[16]. Diffusion tensor imaging (DTI) provides the microstructure changes of brain white matter by measuring the displacement of water molecules across brain tissue components. Fractional anisotropy (FA) obtained from DTI describes the degree of anisotropy of a diffusion process, which can show the treatment effects associated with the changes of specific area of the white matter fiber using voxelwise statistics on the skeletonised white matter tracts [17].

Tuina has been recorded in the classic work of ancient Chinese medicine Huangdi Neijing, which has played an important role in the health of Chinese people for thousands of years. It takes advantage of the hands of the therapist to manipulate the surface of the patient, including relaxation techniques, such as pressing, kneading, etc., which has significant analgesic effect, improving mood, improving patients' confidence in prevention and treatment, and promoting rehabilitation, and reduce the use of health services[18, 19]. Tuina also can relieve the pathological fatigue of muscles, restore the physiological balance of muscles and bones[20], regulate the expression of TNF-a, MMP-13, integrin a1 and integrin $\beta 1$ to inhibit the apoptosis of chondrocytes, promote the metabolism of cartilage extracellular matrix, and inhibit the degeneration of articular cartilage[21]. In addition, a large number of domestic and foreign studies have also shown that education is conducive to the treatment of $\mathrm{KOA}[22,23]$.

Tuina is a promising treatment option for KOA[1], which is based on the theory of meridians and acupoints and modern anatomy. Many studies have shown that Tuina is safe and effective for KOA in 
terms of pain, stiffness, physical function, quality of life, articular cartilage wear, ligament damage and inflammatory response[24-27]. As the well documented effects on pain, Tuina has been recommended for the prevention and cure of KOA by the Chinese guideline and expert consensus[1,28]. Under the guidance of the theory of TCM, doctors mainly use their hands to do the standard Tuina Manipulation on the patient's body surface, which can dredge Channels and Collaterals, activate Qi and Blood circulation, etc., so as to achieve the relief purpose of treating KOA pain and other symptoms[29]. Meanwhile, Tuina treatment could affect the brain plasticity such as structure and functional activity in the patients with chronic pain[14, 15, 30-32].

However, there are few researches on the longitudinal effect and central analgesic mechanism of Tuina in patients with KOA chronic pain, which hinders its wider application in clinic worldwide. The current study aims to (1) investigate the brain alternations in KOA chronic pain patients with combined clinical assessments and structural, DTI and fMRI techniques and (2) explore the longitudinal mechanism of brain plasticity changes after Tuina pain relief treatment for KOA patients compared to health care education treatment.

\section{Methods/design}

\section{Participant recruitment}

All patients with KOA will be recruited from the Tuina department of Yueyang Hospital of Integrated Traditional Chinese and Western medicine, Shanghai University of TCM. Meanwhile, potential patients will also be recruited through posters, the internet and leaflets. In addition, written informed consents will be taken from all patients. A total of 60 patients with KOA chronic pain will be randomly divided into Tuina group and Education group in a 1:1 ratio.

This study protocol has been approved by the Ethics Committee of Yueyang Hospital of Integrated Traditional Chinese and Western Medicine, Shanghai University of TCM. The trial was also registered to the Chinese Clinical Trial Registry.

\section{Study design}

This is a single-center and parallel randomized controlled trial. A total of 60 participants diagnosed with KOA based on American College of Rheumatology (ACR) criteria (1991 revised version)[33] will be considered as eligible patients. They will be randomly allocated into two equal groups with 30 patients in each group, including a Tuina group and a Education group. The interventions of both groups will last for $30 \mathrm{~min}$ and be carried out twice each week. The treatment period will be 12 weeks and the follow-up period will be 48 weeks.

Outcome measurements and MRI scans will be assessed at the baseline, the 6 and 12 weeks after treatment, followed by 48 weeks of clinical follow-up. The changes of clinical variables and cerebral activity of each group will be analyzed after data collection. 
Assessor and analyst will be blinded to the group assignment in the study. Outcome assessment and statistical analyses will be performed by independent researchers who are blinded to the patient assignment. The trial flowchart and study design are shown in Figure 1 and Figure 2, respectively.

\section{Inclusion criteria}

1. meet the diagnostic criteria for KOA set by the ACR in 1991[33]

2. are aged between 40 and 60 years, right-handed, left knee pain

3. have I-II degree knee-joint radiological change on the Kellgren-Lawrence scale

4. have an average knee-pain score on a VAS $\geq 3$ (range from 0 to 10 ) in the past 3 months

5. Volunteer to take part in the study and sign the informed consent form

\section{Exclusion criteria}

1. Are taking analgesics or anesthetics in the past 1 month that may influence brain-imaging outcomes

2. Having received any other treatment in the past 1 month

3. Are pregnant or lactating women

4. Are suffering from mental disease, neurological disease, infectious disease, gastrointestinal disease, cardiovascular and cerebrovascular disease, immunologic disease, respiratory disease or kidney disease, any other chronic pain symptoms, or have a history of brain injury with loss of consciousness

5. Are diagnosed as cancer, tuberculosis, rheumatism or rheumatoid arthritis, gout, joint trauma

6. Have MRI contraindications such as claustrophobia, cardiac pacemaker, defibrillator, heart stent, intrauterine device

7. Have skin lesion around the knee joint

\section{Suspension criteria}

1. Are not suitable to continue to participate in the study due to adverse events or serious adverse events during the trial

2. Are not suitable to continue to participate in the study because of serious deterioration of disease or some complications and special physiological changes

\section{Dropout criteria}

1. withdrew from the study

2. Loss of contact

\section{Sample size}


Sample size is required to compare two group mean values. The calculation method of bilateral equality of two samples is adopted[34] for two groups of $A=$ Tuina group and $B=$ Education group. Suppose H0: $\mu \mathrm{A}-\mu \mathrm{B}=0, \mathrm{H} 1: \mu \mathrm{A}-\mu \mathrm{B} \neq 0$. According to the previous relevant research and the pre experiment results $[24$, 35], we expect the VAS score difference between and after Tuina as $\mu A=3$, and the VAS score difference between and after health care education intervention as $\mu \mathrm{B}=0.7$, if assuming that the standard deviation $\sigma$ of the two groups is equal, that is, $\sigma=2.1$. If we set $\alpha=0.99$ as the type I error, $\beta=0.10$ as the type II error, and $1-\beta=0.90$ as the power, the final result is that we need at least 25 samples in each group.

Many MRI studies reported statistical significance with 12 to 21 patients per group[17, 36-38]. In order to have a powerful and repeatable statistical effect, we require 25 patients per group in this trial. Considering a $20 \%$ dropout rate and the possible excessive head motion during scanning, it will include $30 \mathrm{KOA}$ participants in each group. In summary, we plan to enroll 60 participants and each group will undergo MRI scans to investigate different central mechanisms between Tuina and health care education treatments.

\section{Randomization}

Eligible patients will be randomized in a ratio of 1:1 to the Tuina group and the Education group with 30 patients in each group using simple randomization by the professional responsible for data statistics of the subject. The random number lists will be created will be generated by a random number generator (IBM SPSS Statistics version 21.0 software; IBM Corp., Armonk, NY, USA) and will be sent to the therapist with sequentially numbered, opaque and sealed envelopes by an independent assistant. The therapist will open random-allocation envelopes and allocate the participants accordingly to the Tuina group or the Education group.

\section{Blinding}

Participants and therapists will not be blinded to treatment allocation, due to the limitation of the Tuina treatment and the education intervention. The evaluators, data managers, and statisticians will be blinded to the group allocation in the outcome evaluation procedure and data analysis for the sake of reducing the risk of bias.

\section{Interventions}

The participants will receive a total of 24 treatments in 12 weeks. The doctors must have at least 10 years of experience with Tuina treatment and be skillful in health care education for the participants. In addition, they are required to have passed a clinical test to make sure that the consistency of the trial is administered.

\section{Tuina group}

The selected acupoints for the Tuina treatment include EX-LE02 (heding), EX-LE04 (neixiyan), EX-LE05 (xiyan), GB34 (yanglingquan), SP10 (xuehai), ST34 (liangqiu), ST32 (futu), BL40 (weizhong), BL57 
(chengshan), GB31 (fengshi) and Ashi acupoints around the knee joint. An ashi point is a temporary acupoint where there is a sensation of acid, distension and pain, which can appear anywhere in the knee joints[39].

Referring to previous studies[29, 40-42], the Tuina procedures are as follows: First, the patients are in a supine position. The doctor stands on the side of the patient. The Pressing and Kneading Manipulation of Tuina is applied to the anterior acupoints around the affected knee joint for 10 to $15 \mathrm{~min}$, with the thumb to achieve Deqi sensation, which is commonly regarded as an indicator of manipulation efficacy[43, 44]. Second, the patients are in a prone position. Then the physician will perform the same manipulation on the patient's posterior acupoints around the affected knee joint for 10 to $15 \mathrm{~min}$. It plays the role of relaxing sinews and activating collaterals with the theory of TCM. Every patient will receive $30 \mathrm{~min}$ manipulation each treatment, two treatments per week for 12 weeks.

\section{Education group}

The education intervention will be given to the participants with two sessions per week. The first health education session is a group meeting using a PowerPoint presentation, which will last $30 \mathrm{~min}$. An educational booklet and a video will be given to the patients to do the home-based online e-learning module. The later sessions will be conducted as one-to-one communication focused on personal needs to guide patients to implement the key education in their lives. The program includes two broad components: (1) introduce key concepts of pain biology and (2) present specification of recovery.

Each patient will be given an introduction about the underlying mechanisms, predisposing factors, and prognosis of KOA. Pain will be explained as the conscious part of the response, which can be influenced by many factors as a protective output. Interventionists will formulate specification of daily routines for patients, for example keeping the knees warm, keeping a healthy diet and prevent obesity, maintaining beneficial posture and abandoning bad habitual posture, avoiding overwork, etc.

\section{Concomitant medications and other interventions}

Patients with KOA will be instructed not to take any caffeine (tea and coffee, etc.), any other medications, weight-loss techniques or physical activity, etc. to avoid confound interference for the analgesic effect of intervention of two groups. In cases of severe knee pain, ibuprofen (300 mg per capsule with sustained release) will be allowed as rescue medication and should be recorded on the knee-pain diary. After the end of the study, patients will be taught weight-loss techniques and physical activity. Patients are also asked to record the name, dose, date, frequency and the exact time of the medications used, and to report to the researcher if they take any medications during the study.

\section{Safety evaluation}

Adverse events refer to the unexpected responses that occur during or after treatment, which can lead to a hospitalization or even threaten life. The trial should be suspended and immediate process is indispensable whenever we encounter adverse events. We should record the details in the case report 
forms (CRFs). The efficacy and safety of the intervention will be evaluated after 6,12 weeks' intervention and 48 weeks' follow-up subsequently. In addition, evaluators can be reformed with the clinical symptoms and AEs whenever the patients meet something important.

\section{Outcome measurement}

The clinical outcome will be measured by six self-report questionnaires, which can reflect the pain degree, pain sensation, pain emotion and quality of life.

The primary outcome measurements is VAS[45]. VAS is used to assess the current degree of pain. VAS is a 10-point scale selected to quantitatively measure the level of KOA pain during the study, for which 0 means None-of-Pain while 10 represent the unbearable pain.

The secondary outcomes are SF-MPQ[46], WOMAC[47] and SF-36[48]. SF-MPQ is used to assess pain perception experience and focuses on evaluating the sensory and affective components of knee pain. It mainly consists of 15 descriptors ( 11 sensory and 4 affective) rating on an intensity scale as $0=$ none, 1 = mild, $2=$ moderate, and $3=$ severe. A higher total score indicates more severe pain. The WOMAC is a self-administered questionnaire consisting of 24 items divided into three subscales: pain (5 items), stiffness ( 2 items) and physical function (17 items). These measurements will be used to subsidiarily evaluate the symptom and quality of life improvement. Furthermore, to investigate the influence of emotional state on the brain activity, HAMD[49] and HAMA[50] will be used.

\section{MRI data acquisition}

MRI data will be acquired with a 3.0-T magnetic resonance scanner (SIEMENS MAGNETOM Verio syngo MR B17, Germany) with a 32-channel phase-array head coil at the Medical Imaging Department of Yueyang Hospital of Traditional Chinese and Western Medicine, Shanghai University of TCM. Participants are asked to close their eyes, wear earplugs, keep their heads still throughout the scan, keep relax and stay awake, without thinking of anything particular during the whole scan.

Prior to the blood-oxygen-level-independent (BOLD) resting-state functional images, a high-resolution T1 image for each subject will be acquired with spin echo (SE) sequence, transverse sagittal scan, flip angle $(F A)=9^{\circ}$, pulse repetition time $(T R)=1900 \mathrm{~ms}$, echo time $(T E)=2.93 \mathrm{~ms}$, field of view $(F O V)=256 \times 256$ $\mathrm{mm}^{2}$, slices 160 and slice thickness $=1 \mathrm{~mm}$.

Diffusion tensor imaging will be measured with the axial DTI mapping sequences, $T R=10000 \mathrm{~ms}, \mathrm{TE}=$ $89 \mathrm{~ms}$, matrix $=240 \times 240$, slice thickness $=2 \mathrm{~mm}, \mathrm{~B}$-value $1=0 \mathrm{~s} / \mathrm{mm} 2, \mathrm{~B}-\mathrm{value} 2=1000 \mathrm{~s} / \mathrm{mm} 2,30$ directions.

The BOLD resting-state functional images will be obtained with echo-planar imaging (EPI) sequence as: coronal axial scan, 33 slices, $4 \mathrm{~mm}$ thickness, $\mathrm{TE}=30 \mathrm{~ms}, \mathrm{TR}=2000 \mathrm{~ms}$, FOV $=220 \times 220 \mathrm{~mm}^{2}$, voxel size $=3.4 \times 3.4 \times 4.0 \mathrm{~mm}, \mathrm{FA}=90^{\circ}$, scanning time $8 \mathrm{~min} 8 \mathrm{sec}, 240$ volumes in total. 
The MRI outcome include: structure - gray matter density, cortical thickness, subcortical nuclei volumes; diffusion - FA and MD of white matter integrity; fMRI - functional connectivity (FC).

The 3D T1 structure data analysis will be performed by FSL tools (FMRIB Software Library)[51]. SIENAX (part of FSL 5.0)[52] will be used to obtain the volumes of neocortical gray matter (GM), total GM and white matter (WM) etc. The normalized volumes of subcortical regions such as hippocampus and thalamus will be estimated from FMRIB's integrated registration and segmentation tool (FIRST) (part of FSL 5.0, FMRIB Software Library)[53]. The cortical thickness at each vertex will be obtained using FreeSurfer (http://surfer.nmr.mgh.harvard.edu). DTI data processing pipeline will follow diffusion toolbox in FSL to obtain FA and MD images, and voxewise tract based spatial statistics (TBSS) method will be applied to examine FA/MD changes. For the resting state fMRI data, preprocessing and functional connectivity analysis will be performed by SPM12 software (SPM12, Wellcome Department of Imaging Neuroscience, London, UK; http://www.fil.ion.ucl.ac.uk/spm/) with MATLAB 2013b (Mathworks, Inc., Natick, MA, USA).

\section{Data collection and monitoring}

The screeners will collect data on the baseline characteristics when the patients are recruited. Case report form (CRFs) includes observation and scanning time points, outcome measures, adverse events and safety evaluations. Doctors are blinded to evaluate various clinical pain indicators and fill in relevant information timely and accurately according to the requirements of CRFs. Only outcome assessors can access to the CRFs and perform double-data entry. Then two data administrators, who are beyond the research team and blinded to group allocation, will independently receive the completed CRF and enter them into an Excel database (Microsoft, Redmond, WA, USA). They are required to have completed rigorous training for the data monitoring. Then they will entry the real-time data in the Chinese Clinical Trial Registration Center, in which the electronic data management system will be used to track and monitor the test data in real-time in the Department of Science and Technology in Yueyang Hospital.

\section{Statistical analyses}

When processing and analyzing clinical data, the intention-to-treat principle will be followed with SPSS 21.0 statistical software (IBM, Armonk, New York, USA) by statisticians who are blinded to the group allocation.

The baseline characteristics will be expressed with descriptive statistics for the two groups, which are reported as the mean \pm standard deviation. A Kolmogorov-Smirnov test with Lilliefors correction will be used to analyze all quantitative variables to determine whether they follow a normal distribution.

Parametric statistics (Tukey test) or nonparametric statistics (Wilcoxon rank-sum test) will be used for the within and between-group analyses in accordance with the results of the homogeneity and normality analyses. When initial homogeneity and normality of data distribution are found, repeated measures analysis of variance (ANOVA) will be implemented, or the Friedman test and Kruskal-Wallis test will be used when initial homogeneity but not normality of data distribution is found, or a linear mixed model will 
be adjusted for the baseline value if the initial homogeneity is not found. Adverse events in each group will be documented as percentage for safety assessments using the chi-square test or Fisher's exact test. The statistical significance is defined as $P<0.05$, and the $95 \%$ confidence interval will be reported.

\section{Quality control}

During the processing of the trial, quality control will be conducted with the management of the steering committee. To ensure the consistency of methods, professional trial method and regular monitoring technique should be trained before the researchers participate in the trial. The steering committee and ethics committee should be informed if the study protocol is modified or corrected.

\section{Discussion}

Chronic pain of KOA is not completely consistent with the clinical imaging performance of the knee joint[54], which is very common in clinical diagnosis and treatment, such as there is still a high probability of pain after successful operation[55]. This may be due to the fact that the brain plays a key role as the integration center of neural signals[56]. Reducing joint pain is an important part of KOA treatment recommendations in the international OARSI guidelines (2014)[57] and Chinese Osteoarthritis Diagnosis and Treatment Guidelines (2018)[1].

Tuina belongs to the key noninvasive and non-drug therapies of TCM with distinctive characteristics[58, 59], which has been used for pain relief in KOA for more than a thousand of years in China and some other surrounding countries. Although thalamus is an important part of pain matrix, previous studies have also found abnormal thalamus structure in patients with $\mathrm{KOA}[60]$, but the regulatory mechanism of thalamus and related brain regions involved in Tuina analgesia is still unclear, especially the relationships among different brain tissues and the relationship between different brain tissues and clinical behavioral indicators are still unclear, which limits the promotion and application of Tuina. Therefore, it is necessary to give full play to the advantages of Tuina, evaluate the analgesic effect and central mechanism, so as to better alleviate the pain of KOA.

Multimodal MRI is a cutting-edge technology in the study of pain brain network mapping and analgesia mechanism. It can explore the mechanism of pain, evaluate and predict pain, and is widely used in brain science and pain research. The brain MRI is one of the most commonly used neuroimaging technologies in the study of central mechanism, which has the advantages of its high quality of spatial resolution, no radiation, rapid imaging velocity, non-invasion and affordable price[60-63]. The pathogenesis of chronic pain and persistent pain of KOA are closely related to the subcortical structures and morphological parameters of subcortical nucleus[16]. Diffusion tensor imaging (DTI) can quantitatively measure diffusion tensor and fractional anisotropy (FA) to examine the microstructure and macrostructure of white matter in vivo[64, 65]. fMRI has been widely used in the clinical and theoretical studies of Tuina analgesia for KOA[66], cervical spondylosis[30, 67], lumbar disc herniation[68, 69], low back pain[15], etc. $[40,70-72]$ and to reveal the central mechanism of Tuina analgesia, which is related to the functional changes of amygdala, hypothalamus, nucleus accumbens, hippocampus, cingulate gyrus and other 
areas $[66,70]$. As a main type of $\mathrm{fMRI}$, rest-state $\mathrm{fMRI}$ has the advantage of providing more comprehensive information than task-related fMRI on the functional architecture of the brain[73]. The cross study of this frontier technology and Tuina can better explain the mechanism of analgesia and solve the pain problem.

This clinical trial will not only study the multi-dimensional evaluation of knee osteoarthritis pain, but also evaluate the physical function, quality of life, mental status and so on. Through the combination of clinical behavior and multimodal MRI indicators, such as brain function indicators (FC), brain structure indicators (FA, MD, GMV), brain network properties, to carry out confirmatory and interactive Tuina analgesia research, which can fill the blank of brain pivot analgesia research in Tuina discipline in this field.

In addition, due to the great difference between humans and animals, as well as the particularity of Tuina manipulation, it is difficult to verify the central regulatory mechanism of Tuina intervention on KOA analgesia in animal experiments, and there are too many uncontrollable factors. The application of multimodal MRI to Tuina discipline can clearly explore the brain central characteristics of KOA pain only in clinical trail, comprehensively and deeply analyze the central analgesic regulation mechanism of KOA patients with chronic pain after Tuina, which is a new trend in the future research of Tuina, and also provide a reference experimental paradigm for the cross study of analgesic effect of TCM non-drug therapy.

\section{Study limitations}

In this study, there is an inevitable limitation that it is difficult to control the methodology of blinding during the physical and health care education intervention, which cannot be blinded for the participants and therapists. The blinding quality should be monitored to control the questionnaire measurement credibility, which is composed of the assessors, the administrators, and the data analysts. In summary, this brain MRI trial is designed to investigate the central mechanism of Tuina in the treatment of KOA by comparing that of Education group, and by analyzing the correlation between the cerebral activity changes and clinical variable changes, so as to provide visualization reference for the clinical application of Tuina for KOA management.

\section{Trial status}

This trial is recruiting patients now. Participant recruitment started in Sep. 2020, and is expected to end in Dec. 2022. The version of this protocol is the 1th version and the completion time is 8, Sep. 2020.

\section{Abbreviations}

KOA:knee osteoarthritis; VAS:the Visual analog scale; SF-MPQ:the Short-Form McGill Pain Questionnaire; HAMD:the Hamilton Depression Scale; HAMA:the Hamilton anxiety scale; WOMAC:the Western Ontario and Mcmaster Universities; SF-36:the Short From 36 Survey Questionnaire. 


\section{Declarations}

\section{Acknowledgements}

Not Applicable.

\section{Funding}

This study is financially supported by National Natural Science Foundation of China (81973973), Threeyear Development Plan Project for TCM (ZY(2018-2020)-CCCX-2004-02). The funders have no business with the design and conduct of the trial.

\section{Authors' contributions}

GG planned the study protocol and drafted the manuscript. YK and SZ planned the study protocol and revised the manuscript. QZ carried out the Tuina intervention of patients,and was responsible for generating and distributing the random numbers. ZW carried out the health care education intervention of patients, and recruited and screened eligible participants in the outpatient department and the inpatient department, and participated in designing the outcome measurements and assessing the outcomes. WS was the study coordinator. MF and YC managed the study. All authors read and approved the final manuscript.

\section{Ethics approval and consent to participate}

Ethics approval was requested and approved through the Ethics Committee of the Yueyang Hospital of Integrated Traditional Chinese and Western Medicine, Shanghai University of TCM. Reference number: 2020-039. Informed consent will be obtained from all study participants before starting any data collection by clinical trial communicator. All participants will present their consent in writing.

\section{Consent for publication}

Not applicable.

\section{Competing interests}

The authors declare that they have no competing interests.

\section{References}

1. Joint Surgery Group. Society O, Chinese Medical Association. Guidelines for the Diagnosis and treatment of osteoarthritis (2018 edition).Chinese Journal of Orthopedics. 2015;38(12):705-15.

2. GBD DALYs And HALE Collaborators. Global, regional, and national disability-adjusted life-years (DALYs) for 359 diseases and injuries and healthy life expectancy (HALE) for 195 countries and 
territories, 1990-2017: a systematic analysis for the Global Burden of Disease Study 2017. Lancet. 2018;392(10159):1859-922.

3. Tie, Xiaojia. Zheng rugeng, Zhao Meng, etc. Prevalence of knee osteoarthritis in the middle-aged and elderly in China: a Meta-analysis. Chinese Journal of Tissue Engineering Research. 2018;22(04):650-6.

4. Chen Wei LV, Hongzhi Z, Xiaolin, et al. Design of an epidemiological survey on the prevalence of knee osteoarthritis in middle-aged and elderly people in China. Journal of Hebei Medical University. 2015;36(04):487-90.

5. Tang X, Wang S, Zhan S, et al. The Prevalence of Symptomatic Knee Osteoarthritis in China: Results From the China Health and Retirement Longitudinal Study. Arthritis Rheumatology. 2016;68(03):648-53.

6. Wang Bin X, Dan D, Shengjie, et al. Prevalence and disease burden of knee osteoarthritis in China: a systematic review. Chinese Journal of Evidence-based Medicine. 2018;18(02):134-42.

7. Liu Q, Niu J, Huang J, et al. Knee osteoarthritis and all-cause mortality: the Wuchuan Osteoarthritis Study. Osteoarthritis Cartilage. 2015;23(07):1154-7.

8. Raja SN, Carr DB, Cohen M, et al. The revised International Association for the Study of Pain definition of pain: concepts, challenges, and compromises. Pain, 2020.

9. Peat G, McCarney R, Croft P. Knee pain and osteoarthritis in older adults: a review of community burden and current use of primary health care. Ann Rheum Dis. 2001;60(02):91-7.

10. Reschetniak VK, Kukushkin ML, Gurko NS. The importance of the cortex and subcortical structures of the brain in the perception of acute and chronic pain. Patol Fiziol Eksp Ter, 2014(4):96-110.

11. Hochman JR, Davis AM, Elkayam J, et al. Neuropathic pain symptoms on the modified pain DETECT correlate with signs of central sensitization in knee osteoarthritis. Osteoarthritis Cartilage. 2013;21(09):1236-42.

12. Pujol J, Martínez-Vilavella G, Llorente-Onaindia J, et al. Brain imaging of pain sensitization in patients with knee osteoarthritis. Pain. 2017;158(09):1831.

13. Petersen KK, Arendt-Nielsen L, Finocchietti S, et al. Age Interactions on Pain Sensitization in Patients With Severe Knee Osteoarthritis and Controls. Clin J Pain. 2017;33(12):1081-7.

14. Li H, Wei Z, Siwen L, et al. Progress in the application of functional magnetic resonance in the research of massage. Tianjin Journal of Traditional Chinese Medicine. 2019;36(03):309-12.

15. Wenli T, Wei W, Hongning J, et al. Brain Immediate Analgesia Mechanism of Tuina in Low Back Pain. Chinese Journal of Traditional Medical Traumatology Orthopedics. 2019;27(01):11-6.

16. Mao CP, Bai ZL, Zhang XN, et al. Abnormal Subcortical Brain Morphology in Patients with Knee Osteoarthritis: A Cross-sectional Study. Front Aging Neurosci. 2016;8:3. DOI:10.3389/fnagi.2016.00003.

17. Jian Y, Qipeng S, Kai Z, et al. The effect of Tai Chi practice on brain white matter structure: a diffusion tensor magnetic resonance imaging study. Res Sports Med, 
2019,27(01).DOI:10.1080/15438627.2018.1502184.

18. Li Jishu. FRELAXING TENDONS TO NOURISH ESSENCETUNAACADEMIC THOUGHT TO INTERVENE. INTHE KNEE OSTEOARTHRITIS THERAPY. Ph.D. Nanjing: Nanjing University of Traditional Chinese Medicine; 2014.

19. Deng Baofeng W, Ying Y. Guanfeng. Study on the clinical effect of Tuina therapy for knee osteoarthritis patients. International Journal of Traditional Chinese Medicine. 2017;39(6):512-5.

20. Liu Kunpeng F. min, Jiang Shuyun, et al. Effects of Tuina on the Mechanical Properties of Skeletal Muscle of Four Limbs in Patients with Chronic Fatigue Syndrome. Chinese Journal of Integrated Traditional Western Medicine. 2012;32(05):599-602.

21. Zhu Hongfei L, Yijie F, Wei, et al. Experimental study of imitate-pressing Tuina on knee osteoarthritis in rabbits. Shanghai Journal of Traditional Chinese Medicine. 2012;46(09):72-5.

22. Bezalel T, Carmeli E, Katz-Leurer M. The effect of a group education programme on pain and function through knowledge acquisition and home-based exercise among patients with knee osteoarthritis: $\mathrm{A}$ parallel randomised single-blind clinical trial. Physiotherapy. 2010;96(2):137-43.

23. Haj Hamad W, Sghir M, Guedria M, et al. Contribution of therapeutic education in the treatment of knee osteoarthritis: Preliminary results. Annals of Physical Rehabilitation Medicine. 2016;59:e158. DOI:10.1016/j.rehab.2016.07.352.

24. Zhang Huan Y, Minjian S, Wei, et al. Therapeutic effect of massage on knee osteoarthritis: a metaanalysis. Hainan Medical Journal. 2019;30(07):925-9.

25. Xu Hui X, LianBo. Kang Bingxin, etc. Clinical efficacy and safety of Chinese Tuina in treating knee osteoarthritis Meta-analysis. Shaanxi Journal of Traditional Chinese Medicine. 2019;40(12):180713.

26. Li Y, Chao S, Jing W. Animal experiment of manipulation function in treating athlete's knee joint patellar ligament and articular cartilage. Journal of Wuhan Institute of Physical Education. 2008;42(08):71-4.

27. Xu Q, Chen B, Wang Y, et al. The Effectiveness of Manual Therapy for Relieving Pain, Stiffness, and Dysfunction in Knee Osteoarthritis: A Systematic Review and Meta-Analysis. Pain Physician. 2017;20(04):229-43.

28. Orthopedic Surgery Group, Orthopedic Society Chinese Medical Foundation, Orthopedic Science Expert Committee. Expert Consensus on the ladder treatment of knee osteoarthritis (2018 edition). Chinese Journal of Joint Surgery(Electronic Edition). 2019;13(01):124-30.

29. Fang M, Song BL. Tuina. 10th ed; China Press of Traditional Chinese Medicine: Beijing, 2016;pp. 232-4.

30. Wang Hao Z, Weibin Z, Hui, et al. Effects of Tuina on Pain-Related Brain Area of Chronic Cervical Spondylotic Radiculopathy. Chinese Journal of Basic Medicine in Traditional Chinese Medicine. 2017;23(06):854-7.

31. Wei'an Y, Zhibi S, Li X, et al. Effect of spinal manipulation on brain functional activity inpatients with lumbar disc herniation. Journal of Zhejiang University(Medical Sciences). 2015;44(02):124-30. 
32. Zhang H, Hao W. Li Duoduo, etc. Effects of traditional Chinese tuina on Default Mode Network (DMN) of patients with chronic cervical radicular pain. Journal of Beijing University of Traditional Chinese Medicine. 2014;37(12):845-50.

33. Altman RD. Classification of disease: osteoarthritis. Semin Arthritis Rheum. 1991;20(06):40-7.

34. Chow S, Shao J, Wang H. Sample Size Calculations in Clinical ResearchM. 2nd Ed. Boca Raton: Chapman \& Hall/CRC Biostatistics Series. Library of Congress, 2008: 89-95.

35. Liu Shunchao W. chongmiao, Wu Yaozhi. Clinical study of Tuina on knee joint function of degenerative knee osteoarthritis. Shanghai Journal of Traditional Chinese Medicine. 2014;48(10):62-3.

36. Desmond JE, Glover GH. Estimating sample size in functional MRI (fMRI) neuroimaging studies: Statistical power analyses. J Neurosci Methods. 2002;118(02):115-28.

37. Shim JH, Kim YT, Kim S, et al. Volumetric Reductions of Subcortical Structures and Their Localizations in Alcohol-Dependent Patients. Front Neurol. 2019;10:247. DOI:10.3389/fneur.2019.00247.

38. Rajagopalan V, Yue GH, Pioro EP. Do preprocessing algorithms and statistical models influence voxelbased morphometry (VBM) results in amyotrophic lateral sclerosis patients? A systematic comparison of popular VBM analytical methods. J Magn Reson Imaging. 2014;40(3):662-7.

39. Xue-Lin Z, Wang MY, Gao XY. Exploration on the development of ashi point of all ages. China Journal of Traditional Chinese Medicine Pharmacy. 2018;29(05):1732-8.

40. Zhang Hao. Central Downlink Inhibition and Regulation Mechanism of Analgesia Effect of Tuinia Based on the Theory of Tong Ze Butong. Shanghai: Ph.D, Shanghai University of Traditional Chinese Medicine; 2014.

41. Ai Jian F, Min S, Wuquan, et al. Biomechanical Effects of Jingjin in Knee Disease. Liaoning Journal of Traditional Chinese Medicine. 2015;42(01):66-7.

42. Sun Wuquan. Clinical study on the operation standard of TCM specific manipulation technique in cervical spondylosis. Ph.D. Shanghai: Shanghai University of Traditional Chinese Medicine; 2013.

43. Zhang K, Xu Y, Ding S, et al. Literature study for acupoint selection rule of rheumatoid arthritis treated with acupuncture. Chinese Acupuncture Moxibustion. 2017;37(02):221-4.

44. Chen D, Yang G, Wang F, et al. Discussing the relationship among the ashi point, tender point and myofascial trigger point. Chinese Acupuncture Moxibustion. 2017;37(02):212-4.

45. Wewers ME, Lowe NK. A Critical Review of Visual Analogue Scales in the Measurement of Clinical Phenomena. Res Nurs Health. 1990;13(04):227-36.

46. Melzack R. The short-form McGill pain questionnaire. Pain. 1987;30(02):191-7.

47. BELLAMY N. Validation study of WOMAC: A health status instrument for measuring clinically important patient relevant outcomes to antirheumatic drug therapy in patients with osteoarthritis of the hip and knee. J Rheumatol. 1988;15(12):1833-40. 
48. McHorney CA, Ware JE, Rachel Lu JF, et al. The MOS 36-Item Short-Form Health Survey (SF-36): III. Tests of Data Quality, Scaling Assumptions, and Reliability Across Diverse Patient Groups. Med Care. 1994;32(01):40-66.

49. Bech P, Allerup P, Gram LF. The Hamilton Depression Scale. Acta Psychiatr Scand. 2007;63(03):2909.

50. Gjerris A, Bech P, Jholm B. S, et al. The Hamilton Anxiety Scale: Evaluation of homogeneity and interobserver reliability in patients with depressive disorders. J Affect Disord. 1983;5(02):163-70.

51. Jenkinson M, Beckmann CF, Behrens TE, et al. FSL Neuroimage. 2012;62(2):782-90.

52. Smith SM, Jenkinson M, Woolrich MW, et al. Advances in functional and structural MR image analysis and implementation as FSL. Neuroimage. 2004;23(Suppl 1):208-19.

DOI:10.1016/j.neuroimage.2004.07.051.

53. Patenaude B, Smith SM, Kennedy DN, et al. A Bayesian model of shape and appearance for subcortical brain segmentation. Neuroimage. 2011;56(3):907-22.

54. Bedson J, Croft PR. The discordance between clinical and radiographic knee osteoarthritis: a systematic search and summary of the literature. BMC Musculoskelet Disord, 2008,9:116.DOI:10.1186/1471-2474-9-116.

55. Ke Xueru Z, Li L, Bo, etc. Correlation between postoperative pain inhibition and postoperative chronic pain in patients with knee osteoarthritis after total knee arthroplasty. Chinese Journal of Pain Medicine, 2017,23(09):709-711.

56. Jie Z, Puwei Y, Wulin K, et al. Review of neural mechanism of chronic pain in osteoarthritis. Chinese Journal of Pain Medicine. 2020;26(06):447-50.

57. McAlindon TE, Bannuru RR, Sullivan MC, et al. OARSI guidelines for the non-surgical management of knee osteoarthritis. Osteoarthritis Cartilage. 2014;22(3):363-88.

58. Kong Lingjun G, Guangxin Z, Qingguang, et al. Analysis on development of “Jin Gu Theory"and typical application from Chinese medical tuina. Shanghai Journal of Traditional Chinese Medicine. 2018;52(12):5-8.

59. Guo Guangxin K, Lingjun Z, Qingguang, et al. Discussion on the origin and application of Jingu assessment theory from the perspective of manipulation. hina Journal of Traditional Chinese Medicine Pharmacy. 2019;34(10):4774-7.

60. Guo Guangxin. Central Analgesic Mechanism of Tuina for Knee Osteoarthritis Based MRI Multimodal Technique. Ph.D. Shanghai: Shanghai University of Traditional Chinese Medicine; 2020.

61. Caria A, Sitaram R, Birbaumer N. Real-Time fMRI: A Tool for Local Brain Regulation. Neuroscientist. 2012;18(05):487-501.

62. Huang W, Pach D, Napadow V, et al. Characterizing acupuncture stimuli using brain imaging with fMRI: A systematic review and meta-analysis of the literature. PLoS One. 2012;7(04):e32960.

63. Colombo B, Rocca MA, Messina R, et al. Resting-state fMRI functional connectivity: a new perspective to evaluate pain modulation in migraine? Neurol Sci. 2015;36:41-5. 
DOI:10.1007/s10072-015-2145-x.

64. Bonney PA, Conner AK, Boettcher LB, et al. A Simplified Method of Accurate Postprocessing of Diffusion Tensor Imaging for Use in Brain Tumor Resection. Oper Neurosurg (Hagerstown). 2017;13(1):47-59.

65. Svärd D, Nilsson M, Lampinen B, et al. The effect of white matter hyperintensities on statistical analysis of diffusion tensor imaging in cognitively healthy elderly and prodromal Alzheimer's disease. PLoS One. 2017;12(9):e185239. DOI:10.1371/journal.pone.0185239.

66. Shi Aijun L, Chunlei Wu, Yuan, et al. Regional homogeneity of resting-state brain activity in knee osteoarthritis patients with chronic pain. Journal of Regional Anatomy Operative Surgery. 2017;26(06):419-22.

67. Chen $\mathrm{Y}, \mathrm{Kelei} \mathrm{H}, \mathrm{Chao} \mathrm{L}$, et al. Curative effect of meridian collateral therapy on abnormal brain activity in neck type cervical spondylosis patients. Functional Molecular Medical Imaging(Electronic Edition). 2017;6(03):1233-8.

68. Zhiyong F, Shan Wu, Shuhua L, et al. The Analgesic Effect of Spinal Massage on Lumbar Disc Herniation by Resting State Functional Magnetic Resonance Imaging. Journal of Guangzhou University of Traditional Chinese Medicine. 2016;33(02):262-4.

69. Lai Shuhua, fan Zhiyong, Z, Jiayou, et al. Effect of Rotatory Manipulation on Brain Function in Resting State in Patients with Lumbar Disc Herniation. Journal of New Chinese Medicine, 2017,49(07):96-98.

70. Li Z, Kuiwen S, Xiaochu Z, et al. Pleasure Circuits in the Brain by Press and Knead Weizhong Acupoint. Academic Journal of Shanghai University of Traditional Chinese Medicine. 2008;22(04):51-3.

71. Li Z, Kuiwen S, Xiaochu Z, et al. Functional MRI Studies of Massage Analgesia Modulating within the Human Brain. International Journal of Traditional Chinese Medicine. 2007;29(06):329-32.

72. Xuming $\mathrm{P}$, Zhiyong F, Yong $\mathrm{H}$, et al. Study of FMRI by Single-finger Meditation Pushing Therapy on Source Points of the Gallbladder and Kidney meridian. Journal of Liaoning University of Traditional Chinese Medicine. 2015;17(07):74-8.

73. Shimony, Joshua S,. Stereotactic. et al. Resting-State Blood Oxygen Level-Dependent Functional MRI: A Paradigm Shift in Preoperative Brain Mapping. \& Functional Neurosurgery Official Journal of the World Society for Stereotactic Functional Neurosurgery. 2015;93(6):427-39.

\section{Figures}




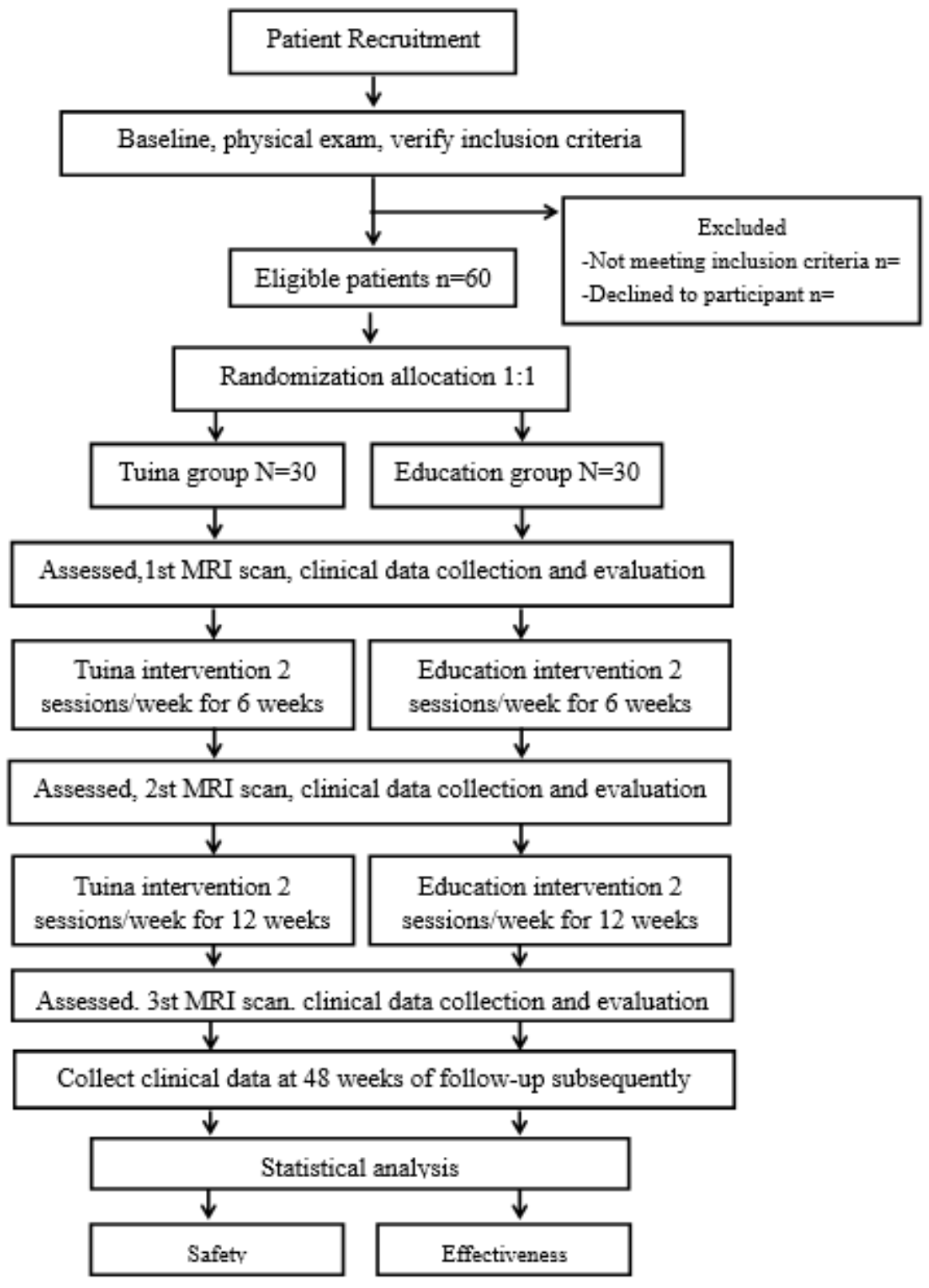

\section{Figure 1}

Flow chart of the trial. The present study is a randomized controlled trial using multimodal MRI. Sixty KOA patients will be included and randomized equally to two groups, a Tuina group and a Education group. For 30 patients in each group, this trial will include a 12-week treatment period. During the treatment, patients in the Tuina group will receive 24 sessions of Tuina treatments, while the Education group will receive health care education. Both the outcome assessments and MRI scan will be performed at 3 time points, namely the baseline, the end of the treatments at 6 weeks and 12 weeks. Only the clinical outcomes will be assessed during 48 weeks of follow-up subsequently. The central mechanism of Tuina in the treatment of KOA will be analyzed after data collection. 


\begin{tabular}{|c|c|c|c|c|c|c|c|}
\hline Time & $-2 W$ & $0 \mathrm{~W}$ & $6 \mathrm{~W}$ & $12 \mathrm{~W}$ & $24 \mathrm{~W}$ & $48 \mathrm{~W}$ & $60 \mathrm{~W}$ \\
\hline Period & Screening & Baseline & \multicolumn{2}{|c|}{ Intervention } & \multicolumn{3}{|c|}{ follow-up } \\
\hline \multicolumn{8}{|l|}{ Enrolment } \\
\hline Eligibility & $x$ & & & & & & \\
\hline Demography & $x$ & & & & & & \\
\hline Informed consent & $x$ & & & & & & \\
\hline $\begin{array}{l}\text { Sign the informed } \\
\text { consent }\end{array}$ & & $x$ & & & & & \\
\hline Medical history & $x$ & & $x$ & $x$ & $x$ & $x$ & $x$ \\
\hline Physical examination & $x$ & & $\mathrm{x}$ & $x$ & $x$ & $\mathrm{x}$ & $x$ \\
\hline Randomization & & $x$ & & & & & \\
\hline \multicolumn{8}{|l|}{ Interventions } \\
\hline $\begin{array}{l}\text { Tuina group } \\
(n=30)\end{array}$ & & & \multicolumn{2}{|c|}{$\begin{array}{l}24 \text { sessions of } \\
\text { tuina }\end{array}$} & & & \\
\hline $\begin{array}{l}\text { Education group } \\
(n=30)\end{array}$ & & & \multicolumn{2}{|c|}{$\begin{array}{l}24 \text { sessions of } \\
\text { education }\end{array}$} & & & \\
\hline \multicolumn{8}{|l|}{ MRI scan } \\
\hline Tuina group $(\mathrm{n}=30)$ & & $x$ & & & $x$ & $\mathrm{x}$ & $x$ \\
\hline Education group $(n=30)$ & & $x$ & & & $x$ & $\mathrm{x}$ & $x$ \\
\hline \multicolumn{8}{|l|}{ Assessments } \\
\hline VAS & & $x$ & & & $x$ & $x$ & $x$ \\
\hline SF-MPQ & & $x$ & & & $x$ & $\mathrm{x}$ & $x$ \\
\hline HAMD & & $x$ & & & $x$ & $\mathrm{x}$ & $x$ \\
\hline HAMA & & $x$ & & & $x$ & $x$ & $x$ \\
\hline WOMAC & & $x$ & & & $x$ & $\mathrm{x}$ & $x$ \\
\hline SF-36 & & $x$ & & & $x$ & $\mathrm{x}$ & $x$ \\
\hline \multicolumn{8}{|l|}{ Trail evaluation } \\
\hline Patients compliance & & & $x$ & $x$ & $x$ & $x$ & $x$ \\
\hline Safety evaluation & & & $x$ & $x$ & $x$ & $\mathrm{x}$ & $x$ \\
\hline Credibility test & & & $x$ & $x$ & $x$ & $\mathrm{x}$ & $x$ \\
\hline Adverse events & & & $x$ & $x$ & $x$ & $\mathrm{x}$ & $x$ \\
\hline Analysis & $x$ & $x$ & $x$ & $x$ & $x$ & $\mathrm{x}$ & $x$ \\
\hline
\end{tabular}

\section{Figure 2}

Study schedule for data collection. The informed consent and examination will be conducted after recruitment. Then, matched KOA patients will be randomized into two groups and receive treatment. Both clinical outcomes and MRI scans will be performed at 3 time points. Only clinical data will be collected at 48 weeks of follow-up subsequently. Adverse events will be recorded in the case report form at any time during the study. VAS Visual analog scale, SF-MPQ the short-form McGill Pain Questionnaire, HAMD Hamilton Depression Scale, HAMA Hamilton anxiety scale, WOMAC the Western Ontario and McMaster Universities Osteoarthritis Index, SF-36 the Short Form 36 Health Survey scale.

\section{Supplementary Files}

This is a list of supplementary files associated with this preprint. Click to download.

- SPIRIT2013Checklist.doc 